\title{
Application of the Sleeping Beauty system in Saanen goat fibroblast cells for establishing persistent transgene expression
}

\author{
B.C. Jiang', H.A. Kaleri ${ }^{1,2}$, H.X. Zhang ${ }^{1}$, J. Chen ${ }^{1}$ and H.L. Liu ${ }^{3}$ \\ ${ }^{1}$ College of Animal Sciences and Technology, \\ Nanjing Agricultural University, Nanjing, China \\ ${ }^{2}$ Department of Animal Breeding and Genetics, \\ Faculty of Animal Husbandry and Veterinary Sciences, \\ Sindh Agriculture University, Tando Jam, Pakistan \\ ${ }^{3}$ Key Laboratory of Animal Genetics, Reproduction, \\ College of Animal Sciences and Technology, \\ Nanjing Agricultural University, China \\ Corresponding author: H.L. Liu \\ E-mail: liuhonglin@263.net
}

Genet. Mol. Res. 10 (4): 3347-3355 (2011)

Received March 25, 2011

Accepted August 9, 2011

Published October 25, 2011

DOI http://dx.doi.org/10.4238/2011.October.25.4

\begin{abstract}
The Sleeping Beauty (SB) transposon system is a promising new method for establishing persistent transgene expression in vivo. We applied the SB system for enhancing transgenesis in Saanen dairy goat fibroblast cells. We constructed a pKT2/CMV-EGFPIRES-PURO vector and investigated the influence of transposon and transposase vector ratios on transfection efficiency in the Saanen goat fibroblast cells. To enhance the SB system performance, we developed a new transfection technique (double-transfection method) for the SB system. The cultured cells were transfected with transposase and transposon vectors successively, with a 42-h interval. Consequently, the transposase and DNA donor (transposon vector) can interact, both at the highest level. Compared with the traditional transfection
\end{abstract}


method, this new double-transfection method approximately doubled integration efficiency.

Key words: Sleeping Beauty system; EGFP; Saanen dairy goat; Fibroblast cell

\section{INTRODUCTION}

Significant effort has been expended to virus-based gene delivery systems (Kovesdi et al., 1997). Viral-vector vehicles, including retroviruses (Gordon and Anderson, 1994), adenovirus (Bruder and Kovesdi, 1997), herpes simplex virus (Latchman, 1994), lentivirus (Naldini, 1998), and adeno-associated virus (Rabinowitz and Samulski, 1998), can dramatically improve the frequency of integration events compared to non-viral vector vehicles. Many attempts have been made to explore a non-viral naked DNA-mediated gene delivery strategy using transposon systems for the expression of new genes in cells and tissues (Dupuy et al., 2002). The Sleeping Beauty (SB) transposon system has been extensively used in animals for transgenesis and has resulted in stable integration and long-term expression of transgenes (Dupuy et al., 2001; Zhu et al., 2010). SB transposon-based transfection consists of a transposase and a transposon. It is active in animal cells, human cultured cells (Ivics et al., 1997), mouse somatic tissues (Yant et al., 2000), and a mouse germ line (Fischer et al., 2001; Horie et al., 2001). It mediates gene transfer that results in precise integration into a TA dinucleotide (Davidson et al., 2009). Transposon integrants mostly express transgenes contained within the vector, unlike proviral integrants that are silenced when passed through the germ line (Jaenisch et al., 1976). Saanen dairy goat is a milking breed and commonly called the poor man's cow (Padeh et al., 1971; Chen et al., 2005). Saanen is among the major candidates for developing bioreactor because of the high level of protein in its milk. Keeping in mind the importance of Saanen dairy goats, this is the first time that the SB system was applied in Saanen dairy goat fibroblasts. We constructed a pKT2/CMVGFP-IRES-PURO vector and established primary cultures of Saanen fibroblasts. The cultured cells were transfected with transposase and transposon vectors using the traditional and the newly developed method (twice-transfection method).

\section{MATERIAL AND METHODS}

\section{Construct preparation}

The CMV promoter and EGFP encoding sequence were amplified from the pEGFP$\mathrm{C} 1$ vector (Clontech). The primers, sense: 5'-GGAAGATCTCCGCCATGCATTAGTTATTA-3' and antisense: 5'-ACATGCATGCTTACTTGTACAGCTCGTCCATG-3', were designed with the enzyme sites $B g l I I$ (5'-end) and $\operatorname{SphI}$ (3'-end). The amplified fragment was inserted in the pKT2/TRE-Tight-BI-GH vector at upstream of EMCV IRES, using the $B g l I I$ and $S p h I$ restriction sites, and the new plasmid was called pKT2/CMV-GFP-IRES-PURO.

\section{Primary culture of Saanen fibroblasts}

Skin biopsies were excised from the ear of a high generic merit 1-month-old Saanen. 
The tissue biopsy was finely cut into small pieces $\left(3-4 \mathrm{~mm}^{2}\right)$ aseptically in phosphatebuffered saline (PBS, GIBCO). The small pieces of tissue explants were cultured in T-75 flasks containing D-MEM (GIBCO) supplemented with 10\% fetal bovine serum (FBS) and antibiotics at $37^{\circ} \mathrm{C}$ in a humidified incubator with $5 \% \mathrm{CO}_{2}$ and $95 \%$ air. After $7-8$ days, fibroblasts appeared attached to the bottom of the flasks, forming a monolayer. When cell confluence was $90 \%$, the explants were removed and the cells were transferred to new flasks. They were cryopreserved in a $-80^{\circ} \mathrm{C}$ freezer before the experiment was performed.

\section{Determining the optimum molar ratio}

Cells at approximately $50 \%$ confluence were treated with medium containing various concentrations of puromycin $(0,1,2,3,4,5 \mu \mathrm{g} / \mathrm{mL})$. The selective media were replenished every 3 days, and the appropriate concentration of puromycin was verified every four days. To obtain the optimum molar ratio for transfection of pKT2/CMV-GFPIRES-PURO to pCMV-SB-Ultra, the pCMV-SB-Ultra and pKT2/CMV-GFP-IRES-PURO mixture was prepared with different molar ratios $(0: 1,1: 100,1: 10,1: 1)$ according to the concentration and molecular weight of vectors.

One day before transfection, fibroblasts were seeded at a density of 5-10 $\times 10^{5}$ per well on 6-well culture plates and cells were $50-70 \%$ confluent at transfection time. First, confluent cells were transduced with different molar ratios of pCMV-SB-Ultra and pKT2/ CMV-GFP-IRES-PURO using lipofectamine 2000, according to the Invitrogen protocol. The medium containing transfection complexes was removed $6 \mathrm{~h}$ later and on the second day after transfection, cells were grown in medium supplemented with $2 \mu \mathrm{g} / \mathrm{mL}$ puromycin. The medium was replaced every 3 days and cells were grown before non-transgenic cells were killed.

\section{Twice-transfection method}

Confluent fibroblast cells (70-90\%) were prepared on 6-well culture plates containing $1.5 \mathrm{~mL}$ D-MEM (GIBCO), 10\% FBS. These cells were randomly divided into three groups.

In group 1 , cells were tranduced with $4 \mu \mathrm{g}$ plasmid mixture of pKT2/CMV-GFPIRES-PURO to pCMV-SB-Ultra (10:1) and after $6 \mathrm{~h}$ the medium with tranfection complexes was removed. Two days after transfection, the cells were grown in medium supplemented with $2 \mu \mathrm{g} / \mathrm{mL}$ puromycin.

In group 2, cells were transduced with $2 \mu \mathrm{g}$ pCMV-SB-Ultra, and after $6 \mathrm{~h}$ the medium with tranfection complexes was removed and a fresh medium was added. The cells were then transduced with pKT2/CMV-GFP-IRES-PURO $42 \mathrm{~h}$ later. Two days after the second transfection, the cells were grown in medium supplemented with $2 \mu \mathrm{g} / \mathrm{mL}$ puromycin.

In group 3, cells were first transduced with $2 \mu \mathrm{g}$ pCMV-SB-Ultra and then $42 \mathrm{~h}$ later with $4 \mu \mathrm{g}$ plasmid mixture of pKT2/CMV-GFP-IRES-PURO and pCMV-SB-Ultra (10:1). Two days after the second transfection, cells were grown in medium supplemented with $2 \mu \mathrm{g} / \mathrm{mL}$ puromycin.

In all groups, the medium was replaced every 3 days and cells were grown until nontransgenic cells were killed. 


\section{Karyotype analysis}

Karyotype analysis of EGFP transgenic fibroblasts was performed with the DAPI Karyotyping kit (GENMED), according to the manufacturer protocol.

\section{Statistical analysis}

The total molecular weight of the DNA molecules pKT2/CMV-GFP-IRES-PURO and pCMV-SB-Ultra was calculated by the MB Advanced DNA Analysis software. Differences were analyzed statistically by one-way ANOVA using the SPSS software, and $\mathrm{P}<0.05$ was considered to be statistically significant.

\section{RESULTS}

\section{pKT2/CMV-EGFP-IRES-PURO vector}

The pKT2/CMV-EGFP-IRES-PURO vector was successfully constructed. The construction map of the newly constructed plasmid pKT2/CMV-EGFP-IRES-PURO is given in Figure 1. Figure 1A provides information about the vector backbone from pKT2/TET-ON$\mathrm{GH}$, showing that the size of the vector backbone is $6549 \mathrm{bp}$. Figure 1B displays the agarose gel electrophoresis of pKT2/TET-ON-GH and (CMV/EGFP) digested with BglII and SphI enzymes, revealing the vector backbone (4668 and $1881 \mathrm{bp}$ ), and CMV/EGFP fragment (1348 bp), compared to a 500-bp ladder marker (Takara). Figure 1C shows the construction map of the pKT2/CMV-EGFP-IRES-PURO vector with a size of $6021 \mathrm{bp}$. Figure 1D shows the digestion result for the pKT2/CMV-EGFP-IRES-PURO vector with NheI and BamHI restriction enzymatic sites, yielding 4442- and 1579-bp fragments, respectively.

\section{The optimum molar ratio for the SB system}

The application of the MB Advanced DNA Analysis software for determining the total molecular weight of DNA molecules of pKT2/CMV-GFP-IRES-PURO and pCMV-SB-Ultra resulted in 3650659 and $2873616 \mathrm{~g} / \mathrm{mol}$, respectively. Figure $2 \mathrm{~A}$ and B shows the molecular weight of DNA molecules of pKT2/CMV-GFP-IRES-PURO and pCMV-SB-Ultra.

Using UV microscopy, GFP was clearly detected in the primary goat cells transfected with the transposase vector and transposon vector but not in cells transfected with only transposon vector. The level of GFP expression was highest in Panel (1:10) compared to $(0: 1),(1: 100)$ and $(1: 1)$ (Figure 3$)$.

The optimal molar ratio of the transposase vector and transposon vector was around 1:10 (Figure 4). The molar ratio had a profound effect on the detection of GFP in cells; a low $(1: 100)$ and high (1:1) molar ratio of transposase to transposon vector yielded fewer positive GFP cells.

\section{Comparison between the traditional and the new method}

The results for comparison between the traditional and the new transfection method 
A

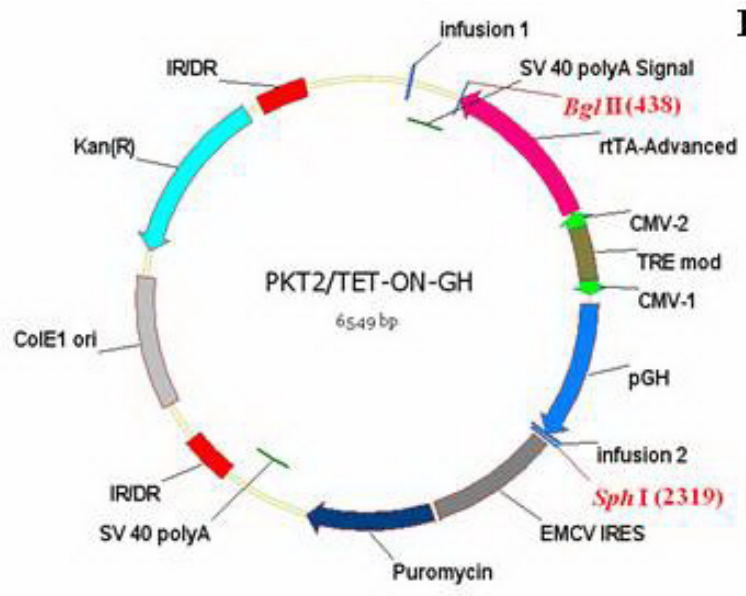

$\mathrm{C}$

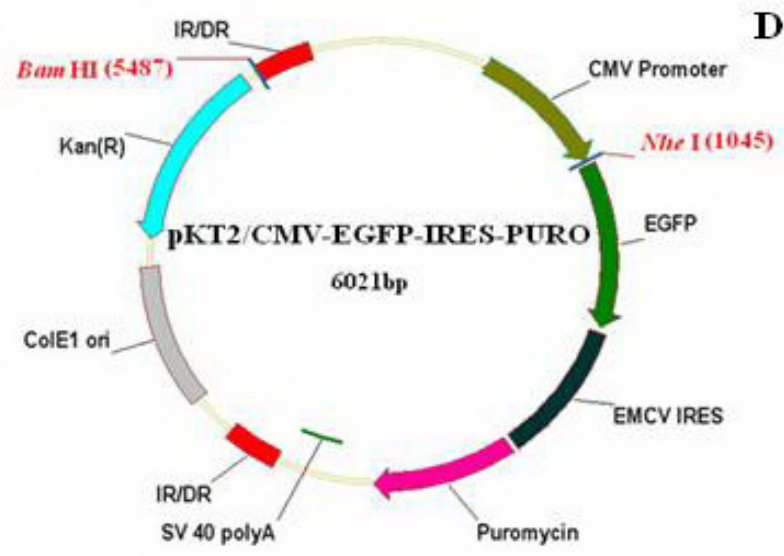

B

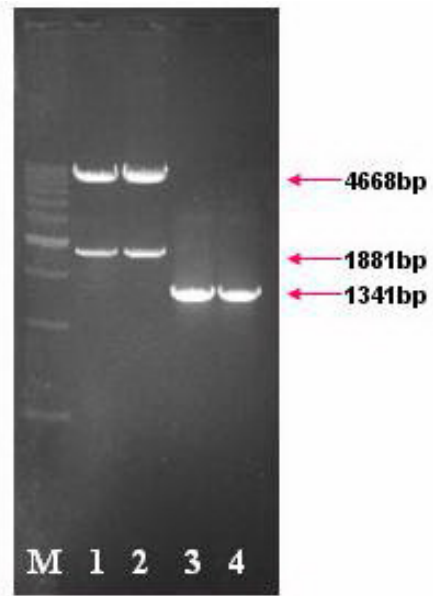

(500kb ladder marker)

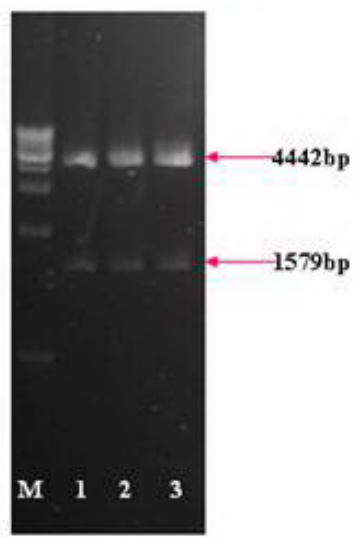

Figure 1. The construction map of the pKT2/CMV-EGFP-IRES-PURO vector. A. Information of vector backbone from pKT2/TET-ON-GH (size 6549 bp). B. Agarose gel electrophoresis of pKT2/TET-ON-GH and CMV/EGFP digested with $B g l \mathrm{II}$ and $S p h \mathrm{I}$ enzymes. Lanes 1 and $2=$ vector backbone; lanes 3 and $4=\mathrm{CMV} / \mathrm{EGFP}$ fragment; lane $M=500$-bp ladder marker (Takara). C. The construction map of the pKT2/CMV-EGFP-IRES-PURO vector (size 6021 bp). D. Digestion result of pKT2/CMV-EGFP-IRES-PURO vector with NheI and BamHI restriction sites. Lanes 1, 2 and 3 = digestion samples (4442 and $1579 \mathrm{bp);} \mathrm{lane} M=1-\mathrm{kb}$ ladder marker (Takara).

are given in Figure 5, which revealed that the newly developed method brought forth more GFP-positive cells. Figure 5 shows the comparison between the traditional and the new method.

\section{Karyotyping}

The karyotype results for GFP-positive cells by chromosomal DAPI-band analysis revealed that these cells had a normal karyotype after being subcultured for 6 passages. Figure 6 shows the chromosomal analysis. GFP-positive cells displayed no changes in chromosome number or morphology. This demonstrated that SB-mediated transfection by the twice-transfection method did not lead to chromosomal abnormalities. 

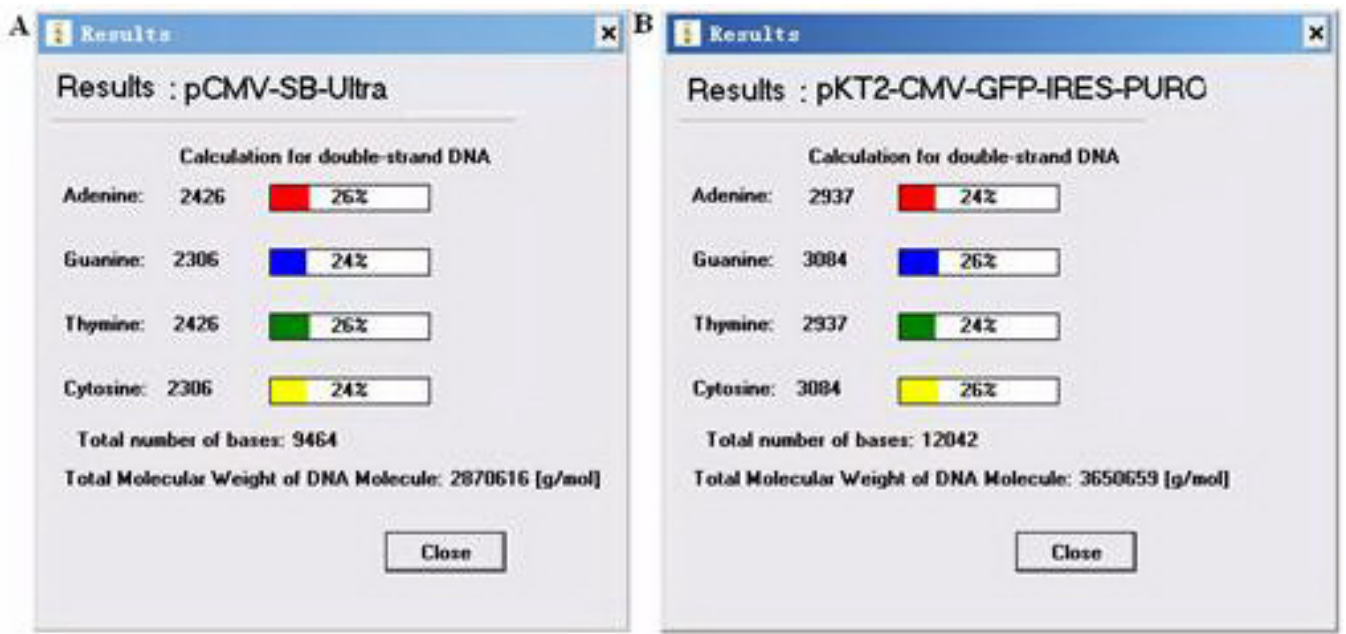

Figure 2. Total molecular weight of DNA molecules of pCMV-SB-Ultra and pKT2/CMV-GFP-IRES-PURO.

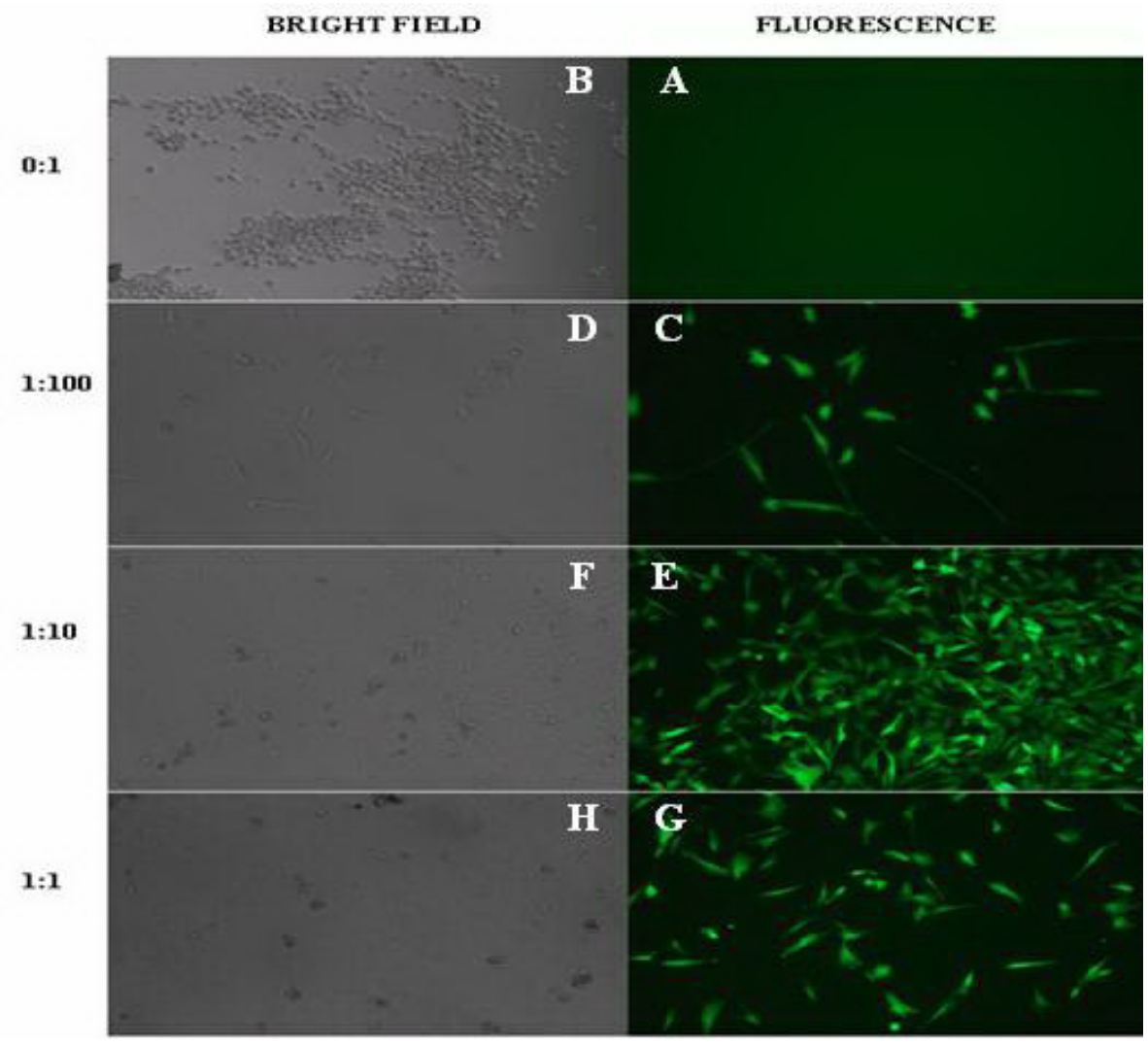

Figure 3. GFP expression in fibroblasts. A. C. E. and G. Fluorescence image. B. D. F. and H. Bright field corresponding to $\mathrm{A}, \mathrm{B}, \mathrm{C}$, and $\mathrm{D}$. 


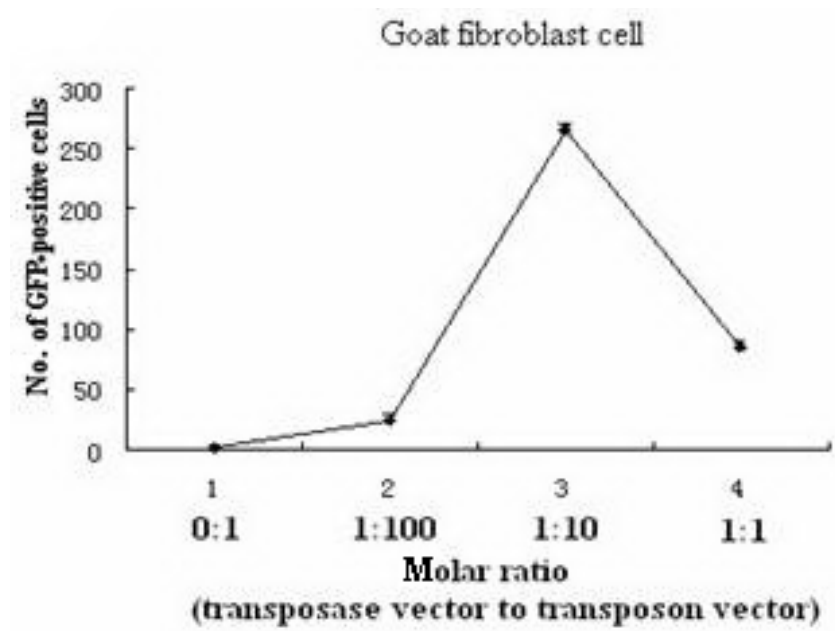

Figure 4. Molar ratio of transposase vector to transposon vector, showing the number of GFP-positive cells.

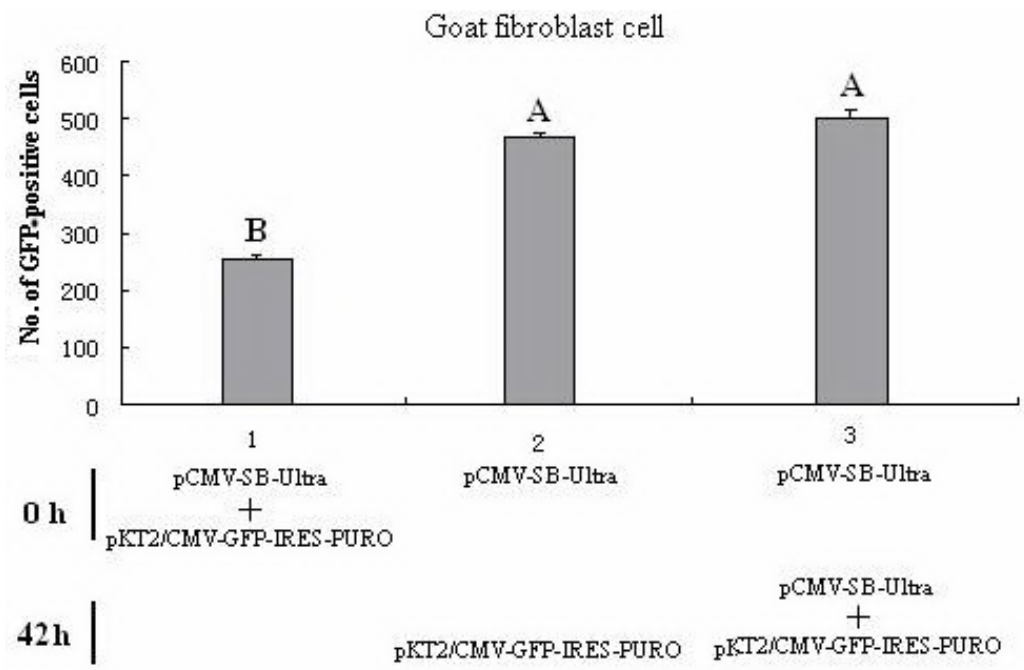

Figure 5. Comparison between traditional and newly developed method (twice transfection method). $1=$ Traditional method of transfection. 2 = Newly developed method (twice transfection method). 3 = Transfection of transposase vector twice and transposan vector once.

\section{DISCUSSION}

The construction of the pKT2/CMV-EGFP-IRES-PURO vector was successful with the insertion of a CMV promoter and EGFP into pKT2/TRE-Tight-BI-GH-rtTA-Advanced in place of the IRES fragment. pKT2/CMV-EGFP-IRES-PURO contains all features of the original vector, including the SB transposase-recognized sites IR/DR. The agarose gel result for size revealed that vector size was $6021 \mathrm{bp}$ and that NheI and BamHI enzymes produced 4442- and 1579-bp fragments, respectively. 


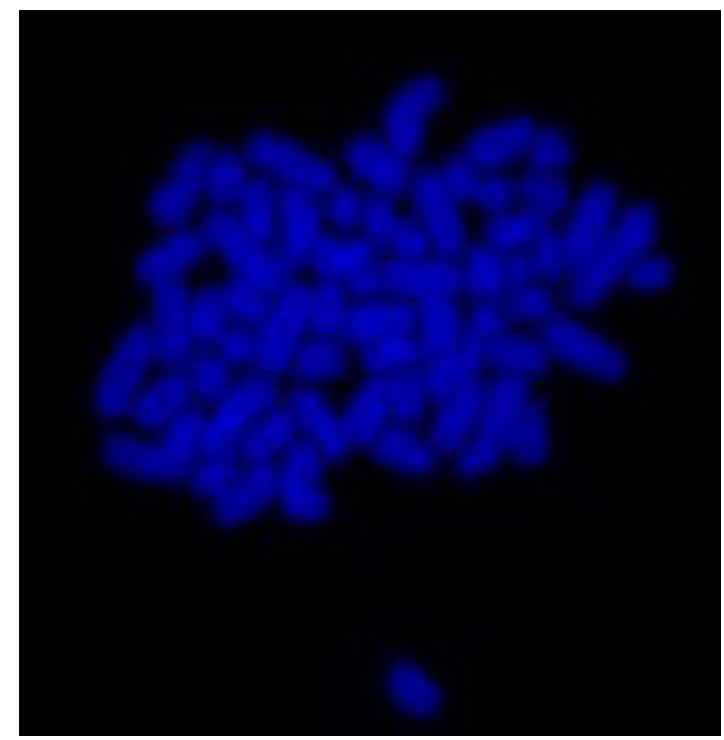

Figure 6. Karyotype analysis of a colony of cells submitted to twice-transfection method (400X magnification).

The results for transfection with transposase and transposon vectors showed that the Sleeping Beauty system can be applied in goat fibroblasts. The expression of the vector remained at peak level after 12 to $72 \mathrm{~h}$ of transfection (Patterson and Lippincott-Schwartz, 2002). We found that a 1:10 molar ratio of transposase to transposon vector was the best for integration efficiency in fibroblast cells, whereas with a 1:100 molar ratio, the transposase level is too low and affects the integration efficiency in fibroblast cells. It was also found that with a 1:1 molar ratio, although the amount of transposase vector is high, the mature proteins were not enough because most of the proteins in cells remain at a premature stage. In the traditional method, we found several conspicuous merits. It is also well known that the level of recombinant protein production is increased at 24-72 $\mathrm{h}$ after transfection (Thierry et al., 1997; Li et al., 2002). When the expression level of the SB transposase protein is high, it becomes difficult to obtain a high level of plasmid DNA because of degradation by normal endosomal pathways (Cole et al., 1992). The level of transposase will continuously decrease because of the competition between the expression of recombinant protein from transposon and transposase vectors. Lipofectamine 2000 capacity for carrying DNA into cells is limited (Thierry et al., 1997), so a small amount of transposase plasmid can enter cells due to an appropriate proportion of transposase and transposon in the mixture. In view of the foregoing, we transduced the SB system into cells twice with a 42-h interval between the transposase and transposon vector transfections. The transposase and DNA donor (transposon vector) interact both at the topmost level, and the recombinant protein from transposon vector cannot be expressed when the transposase is highly active. As the transposase and transposon vectors were transfected separately in our newly developed method, there was a greater chance of their expression as mature rather than immature protein in cells. Thus, we were successful enough to show a higher level of pKT2/ CMV-EGFP-IRES-PURO integration efficiency using the Sleeping Beauty system in goat fibroblasts. We also found that after using our method (twice transfection method), no change was seen in the chromosome set up of cells (Figure 6). 
We conclude that in comparison with the traditional method, the twice transfection method increased the integration efficiency to a maximal level. Thus, this is the first successful attempt to apply the SB system by a new twice-transfection method in goat fibroblasts. Further study is needed to determine the optimal interval time and transfection reagents with lower cytotoxicity.

\section{REFERENCES}

Bruder JT and Kovesdi I (1997). Adenovirus infection stimulates the Raf/MAPK signaling pathway and induces interleukin-8 expression. J. Virol. 71: 398-404.

Chen H, Lan XY, Li RB, Lei CZ, et al. (2005). The effect of CSN1 S2, CSN3 and beta-lg genes on milk performance in Xinong Saanen dairy goat. Yi Chuan Xue Bao 32: 804-810.

Cole GM, Bell L, Truong QB and Saitoh T (1992). An endosomal-lysosomal pathway for degradation of amyloid precursor protein. Ann. N. Y. Acad. Sci. 674: 103-117.

Davidson AE, Gratsch TE, Morell MH, O'Shea KS, et al. (2009). Use of the Sleeping Beauty transposon system for stable gene expression in mouse embryonic stem cells. Cold Spring Harb. Protoc. 2009: db.

Dupuy AJ, Fritz S and Largaespada DA (2001). Transposition and gene disruption in the male germline of the mouse. Genesis 30: 82-88.

Dupuy AJ, Clark K, Carlson CM, Fritz S, et al. (2002). Mammalian germ-line transgenesis by transposition. Proc. Natl. Acad. Sci. U. S. A. 99: 4495-4499.

Fischer SE, Wienholds E and Plasterk RH (2001). Regulated transposition of a fish transposon in the mouse germ line. Proc. Natl. Acad. Sci. U. S. A. 98: 6759-6764.

Gordon EM and Anderson WF (1994). Gene therapy using retroviral vectors. Curr. Opin. Biotechnol. 5: 611-616.

Horie K, Kuroiwa A, Ikawa M, Okabe M, et al. (2001). Efficient chromosomal transposition of a Tc1/mariner- like transposon Sleeping Beauty in mice. Proc. Natl. Acad. Sci. U. S. A. 98: 9191-9196.

Ivics Z, Hackett PB, Plasterk RH and Izsvak Z (1997). Molecular reconstruction of Sleeping Beauty, a Tc1-like transposon from fish, and its transposition in human cells. Cell 91: 501-510.

Jaenisch R, Dausman J, Cox V and Fan H (1976). Infection of developing mouse embryos with murine leukemia virus: tissue specificity and genetic transmission of the virus. Hamatol. Bluttransfus. 19: 341-356.

Kovesdi I, Brough DE, Bruder JT and Wickham TJ (1997). Adenoviral vectors for gene transfer. Curr. Opin. Biotechnol. 8: 583-589.

Latchman DS (1994). Herpes simplex virus vectors for gene therapy. Mol. Biotechnol. 2: 179-195.

Li Y, Wu KF, Guo XD, Guo JT, et al. (2002). Optimization of parameters of exogene transfection of bovine fetal fibroblasts in vitro mediated by liposome. Yi Chuan 24: 653-655.

Naldini L (1998). Lentiviruses as gene transfer agents for delivery to non-dividing cells. Curr. Opin. Biotechnol. 9: 457463.

Padeh B, Wyskoki M and Soller M (1971). Further studies on a Robertsonian translocation in the Saanen dairy goat. Cytogenetics 10: 61-69.

Patterson GH and Lippincott-Schwartz J (2002). A photoactivatable GFP for selective photolabeling of proteins and cells. Science 297: 1873-1877.

Rabinowitz JE and Samulski J (1998). Adeno-associated virus expression systems for gene transfer. Curr. Opin. Biotechnol. 9: 470-475.

Thierry AR, Rabinovich P, Peng B, Mahan LC, et al. (1997). Characterization of liposome-mediated gene delivery: expression, stability and pharmacokinetics of plasmid DNA. Gene Ther. 4: 226-237.

Yant SR, Meuse L, Chiu W, Ivics Z, et al. (2000). Somatic integration and long-term transgene expression in normal and haemophilic mice using a DNA transposon system. Nat. Genet. 25: 35-41.

Zhu J, Park CW, Sjeklocha L, Kren BT, et al. (2010). High-level genomic integration, epigenetic changes, and expression of sleeping beauty transgene. Biochemistry 49: 1507-1521. 